\title{
Subcomplementary code pairs: New codes for ST/MST radar observations
}

\author{
$\operatorname{AUTHOR}(\mathrm{S})$ :
}

Ghebrebrhan, O; Luce, H; Yamamoto, M; Fukao, S

\section{CITATION:}

Ghebrebrhan, O ... [et al]. Subcomplementary code pairs: New codes for ST/MST radar observations. IEEE TRANSACTIONS ON GEOSCIENCE AND REMOTE SENSING 2003, 41(1): 111-122

\section{ISSUE DATE:}

2003-01

URL:

http://hdl.handle.net/2433/50440

\section{RIGHT:}

(c)2003 IEEE. Personal use of this material is permitted. However, permission to reprint/republish this material for advertising or promotional purposes or for creating new collective works for resale or redistribution to servers or lists, or to reuse any copyrighted component of this work in other works must be obtained from the IEEE. 


\title{
Subcomplementary Code Pairs: New Codes for ST/MST Radar Observations
}

\author{
Ogubazghi Ghebrebrhan, Hubert Luce, Mamoru Yamamoto, and Shoichiro Fukao
}

\begin{abstract}
A new type of codes, named subcomplementary codes, are introduced. These codes are close to, but not strictly, complementary. Each of the two sequences of the pair has an equal number of opposite elements, which enables the codes to have very high interference-suppression-factor (ISF) performances in and around the radar center frequency. The disadvantage of these codes is the presence of sidelobes of amplitude of $-N$ in their autocorrelation functions for lag 1 ( $N$ being the code length). Some properties of these codes are presented along with a technique for generating the code pairs. Subcomplementary code pairs have been found for values of $N$ equal to $4,8,16,20$, and 32. A simulation study confirms a major improvement in ISF over complementary code pairs around the zero Doppler frequency. Experimental observations were performed with the middle and upper atmosphere radar in Japan using complementary and subcomplementary code pairs of length 16 and an uncoded pulse for range resolution performance comparisons. The results obtained so far indicate that the effects of the sidelobes in the subcomplementary code pair are minimal for wind observations, although significant for shear velocity observations. The degradation in performance in signal-to-noise ratio observations is found to be noticeable but not severe. The subcomplementary code pairs may, therefore, be used in situations where their advantages for interference suppression are exploited and where the effects of their weaknesses are not so important as in the case of observations for applications in meteorology.
\end{abstract}

Index Terms-Complementary code pairs, interference suppression factor, radar decoding, stratosphere-troposphere radar.

\section{INTRODUCTION}

$\mathbf{C}$ OMPLEMENTARY code pairs have been used in stratosphere-troposphere/mesosphere-stratosphere-troposphere (ST/MST) radar systems for over 20 years [1]-[3]. They are used to maintain the duty cycle of the radar transmitter at its optimum value despite high-altitude resolution requirements that would otherwise reduce it to lower levels [4]. A number of studies have been conducted by users of the codes to address some of the inconveniences resulting from imperfections originating from atmospheric characteristics [3]-[5], instrumental imperfections [6], [7], and the truncation effects of the codes [8]-[10].

Manuscript received August 12, 2001; revised August 25, 2002. This work was done while the first author was a visiting professor at the Radio Science Center for Space and Atmosphere (RASC) and H. L. was a Japan Society for Promotion of Science postdoctoral fellow at RASC. The MU radar belongs to and is operated by RASC, Kyoto University.

O. Ghebrebrhan is with the Radio Science Center for Space and Atmosphere (RASC), Kyoto University, Kyoto 611-0011, Japan, and also with the Department of Earth Sciences, University of Asmara, Asmara, Eritrea.

H. Luce, M. Yamamoto, and S. Fukao are with the Radio Science Center for Space and Atmosphere (RASC), Kyoto University, Kyoto 611-0011, Japan.

Digital Object Identifier 10.1109/TGRS.2002.807752
In addition to their role in enhancing range resolution performances, complementary codes also possess the capacity to suppress interference signals not coded by the radar system. The principle involved here is that while the signals that have been coded by the radar (that includes the geophysical signal which is the desired signal) are coherently decoded by the decoding system, the interference signals are decoded incoherently [9], [11]. This aspect of the codes has recently been studied in more detail, and a parameter called the interference suppression factor (ISF) has been defined [12].

The characteristics of the ISF of complementary codes depend, among other things, on the composition of the elements of the codes. Better results are obtained using new codes that are close to, but not strictly, complementary. In this paper, the nature of these codes and their ISF characteristics, as well as the technique for their generation, are presented. Experimental observations aimed at testing their resolution performance are also included.

The paper is organized in the following manner. After a brief review of complementary codes and their ISF characteristics, some details of the new codes, the subcomplementary code pairs, as well as their interference suppression capabilities, are presented. This is followed by a brief description of the technique for generating these code pairs. Finally, the details of the experimental observations and the concluding section are given.

\section{COMPLEMENTARY CODES}

\section{A. Complementary Code Pairs}

A complementary code pair consists of two code sequences of equal length. The two code sequences $C_{0}$ and $C_{1}$ may be represented as [13]

$$
\begin{aligned}
& C_{0}=\left\{c_{1}, c_{2}, \ldots c_{N}\right\} \\
& C_{1}=\left\{c_{1}^{\prime}, c_{2}^{\prime}, \ldots c_{N}^{\prime}\right\}
\end{aligned}
$$

such that

$$
c_{i}, c_{i}^{\prime} \in\{1,-1\}, \quad i=1,2, \ldots, N
$$

where $N$ is the number of elements of each sequence.

Complementary code pairs have the important property that

$$
R_{0}(\gamma)+R_{1}(\gamma)= \begin{cases}2 N, & \text { if } \gamma=0 \\ 0, & \text { otherwise }\end{cases}
$$


where $R_{0}$ and $R_{1}$ are the autocorrelation functions (ACFs) of $C_{0}$ and $C_{1}$, respectively, and are given by

$$
R_{0}(\gamma)=\sum_{i=1}^{N-\gamma} c_{i} c_{i+\gamma}
$$

and

$$
R_{1}(\gamma)=\sum_{i=1}^{N-\gamma} c_{i}^{\prime} c_{i+\gamma}^{\prime}
$$

In a radar system using complementary code pairs, two consecutive pulses $P_{o}$ and $P_{1}$ are coded, respectively, by code sequences $C_{0}$ and $C_{1}$. The decoding process involves correlating the received and sampled signals with their corresponding code sequences. That is, the returns from pulse $P_{0}$ are decoded using code sequence $C_{0}$, and similarly the returns from $P_{1}$ are decoded using $C_{1}$ (see [8] and [15] for more details).

The complementary property may be viewed from another angle [16]. As implied by (3), in this paper we are dealing with binary codes, where the elements of the sequences are either 1 or -1 . Following Golay [13], we define an even pair of a given spacing as a pair of identical elements (like $\{11\}$ or $\{-1-1\}$ ) and odd pairs as the corresponding pairs of unlike elements (like $\{-11\}$ or $\{1-1\})$. As an example, in the sequence $\{-111-1\}$, there are two odd and one even pair with spacing of one, two odd pairs with a spacing of two, and one even pair with a spacing of three. This is illustrated in Fig. 1.

With this background, we represent the total number of even pairs of all spacings of $C_{0}$ and $C_{1}$, by $N_{e 0}^{T}$ and $N_{e 1}^{T}$, respectively. The corresponding expressions are given by

$$
\begin{aligned}
& N_{e 0}^{T}=\sum_{\gamma=1}^{N-1} N_{e 0}(\gamma) \\
& N_{e 1}^{T}=\sum_{\gamma=1}^{N-1} N_{e 1}(\gamma)
\end{aligned}
$$

where

$$
N_{e 0}(\gamma)=\sum_{i=1}^{N-\gamma}\left(0.5 c_{i}+0.5 c_{i+\gamma}\right)^{2}, \quad 1 \leq \gamma \leq N-1 .
$$

The expression for $N_{e 1}(\gamma)$ is similar to that of $N_{e 0}(\gamma)$, except that $c_{i}^{\prime}$ is used in place of $c_{i}$. Similarly, $N_{o 0}^{T}$ and $N_{o 1}^{T}$ are defined as the total number of odd pairs of all spacings in $C_{0}$ and $C_{1}$, respectively. The expressions for $N_{o 0}$ and $N_{o 1}$ have the form

$$
N_{o 0}(\gamma)=\sum_{i=1}^{N-\gamma}\left(0.5 c_{i}-0.5 c_{i+\gamma}\right)^{2}, \quad 1 \leq \gamma \leq N-1 .
$$

The expression for $N_{o 1}(\gamma)$ is similar to that of $N_{o 0}(\gamma)$ except that $c_{i}^{\prime}$ is used in place of $c_{i}$. With this notation, $C_{0}$ and $C_{1}$ are complementary if and only if

$$
N_{e 0}^{T}=N_{o 1}^{T}
$$

or

$$
N_{o 0}^{T}=N_{e 1}^{T} .
$$

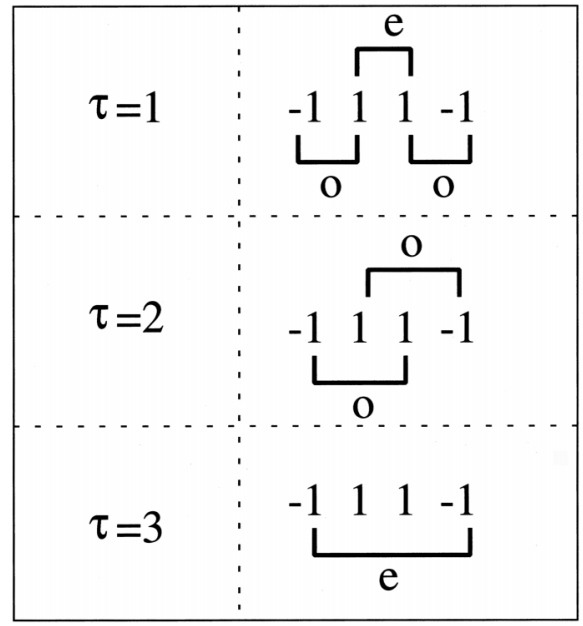

Fig. 1. Example for the identification of pairs with spacings of 1,2, and 3. The letters " $e$ " and " $o$ " stand for even and odd pairs, respectively.

It can easily be verified that the two equations are not independent.

This definition and the one based on the autocorrelation function [see(4)] can be related by

$$
\begin{aligned}
& R_{0}(\gamma)=N_{e 0}(\gamma)-N_{o 0}(\gamma) \\
& R_{1}(\gamma)=N_{e 1}(\gamma)-N_{o 1}(\gamma)
\end{aligned}
$$

Using the relationships stated by (11) or (12), Golay [13] derived a necessary but not sufficient condition for complementarity, relating $p$ and $q$, the number of ones in sequences $C_{0}$ and $C_{1}$, respectively. This condition is given by

$$
N=(N-p-q)^{2}+(p-q)^{2} .
$$

These results are used in Sections III-V.

\section{B. $I S F$}

The radar decoding system decodes the radar returns coherently, whereas signals that have not been coded are decoded incoherently. Accordingly, while the received and sampled signals that have been coded by the radar system pass the decoding system with little or no attenuation, the other group of signals may be attenuated (relative to the geophysical signal) by varying degrees depending on the frequency spectrum of the signals and the codes used. In the second group of signals, we find the following:

1) noise, both internal (electronic or receiver noise) and external (cosmic noise)

2) electromagnetic noise (noise from electric switches, power lines, etc.);

3) various types of radio interference signals (radio, television, etc.).

These are the signals that we are addressing in this paper and for which the decoding process has the potential to attenuate. In order to quantify the degree of attenuation, use is made of the ISF. This is defined in the following manner. A geophysical signal and an interference signal are considered. The corresponding sampled powers in each range gate are assumed to have the powers of $P_{i g}$ and $P_{i i}$, respectively. For the purpose of this definition, $P_{i g}$ is arbitrarily set equal to $P_{i i}$, without loss of generality. After coherent integration and decoding, the powers 
in each range gate of the interference and the geophysical signals become $P_{o i}$ and $P_{o g}$, respectively. Since $P_{i g}$ is assumed to be equal to $P_{i i}$, the ISF (in decibels) is then defined as

$$
\text { ISF }=10 \log _{10}\left(\frac{P_{o i}}{P_{o g}}\right) .
$$

ISF may also be viewed as the improvement in the signal-tointerference ratio after the coherent integration and the decoding processes. We illustrate this using a simple example. Consider geophysical and interference signals where the magnitude of the amplitudes are constant for at least two interpulse periods (this may approximate very low frequency signals). However, as the geophysical signal has been coded by the radar system, its phase varies in accordance with the given code. Thus, the complex values of the signals may be represented as $\tilde{a}$, for the geophysical signal, and as $\tilde{i}$ for the interference signal, with $|\tilde{a}|=|\tilde{i}|$, where the $\operatorname{sign}^{\sim}$ on top of $a$ indicates that it is a complex variable, and with

$$
P_{i i}=P_{i g}=P_{a}=\tilde{i \tilde{i}^{*}}=\tilde{a} \tilde{a}^{*} .
$$

Under this condition, and applying the normal procedures of sampling and decoding, it can be shown that the expression of the ISF is given as [8], [12]

$$
\mathrm{ISF}=10 \log _{10}\left(\frac{N_{s}^{2}}{4 N^{2}}\right)
$$

where $N_{s}$ is given by

$$
N_{s}=\sum_{i=1}^{N}\left(c_{i}+c_{i}^{\prime}\right) .
$$

For an example of this, we consider the case of the code pair of length 16 with sequences of $C_{0}=\{166742\}$ and $C_{1}=$ $\{166435\}$. For ease of representation, code sequences are represented (in what follows) in octal form (obtained from the binary sequence when -1 is replaced by zero). For this code pair, $N_{s}=8$ and ISF $=-12.04 \mathrm{~dB}$.

For some code pairs, $N_{s}$ is zero, and these codes are good in suppressing dc and low (Doppler) frequency interference signals.

For a more general expression of the ISF, we consider a sinusoidal interference signal of the form

$$
\tilde{i}(t)=|\tilde{i}| \exp (j \omega t) \text {. }
$$

Applying the operations of sampling and decoding to this interference signal, the expression for the power of the signal after decoding may be put in the form

$$
P_{o i}=\beta\left(\omega, C_{0}, C_{1}\right) P_{a}
$$

where $C_{0}$ and $C_{1}$ are the sequences of the code pair [see (1) and (2)], and $P_{a}$ is as given by (17). Accordingly, we have

$$
\mathrm{ISF}=10 \log _{10}\left(\frac{\beta\left(\omega, C_{0}, C_{1}\right)}{4 N^{2}}\right) .
$$

The analytical expression for the function $\beta\left(\omega, C_{0}, C_{1}\right)$ has been derived in [15] and has a rather complicated expression that limits its practical utility. Better insight is obtained through numerical simulation. This is done by simulating the sampling and decoding processes as well as the determination of the power in exactly the same manner as the radar signal processor. For an example of this, we perform the simulation for the code

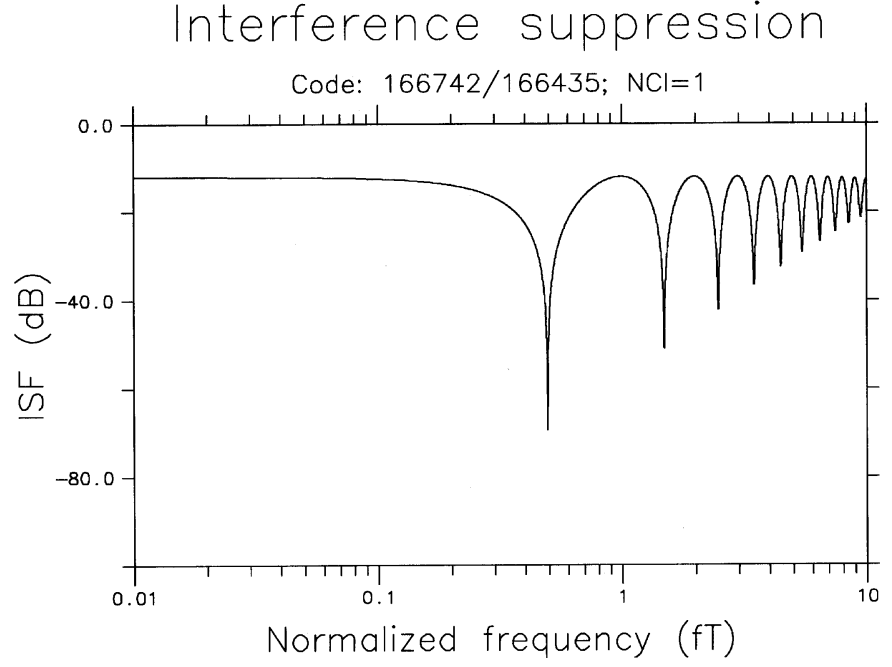

Fig. 2. ISF simulation for complementary code pair of length 16 with sequences of $C_{0}=\{166742\}$ and $C_{1}=\{166435\}$. The sequences are represented in octal format (see text for explanation). NCI $=1$ implies that no coherent integration was performed during the simulation.

pair of length 16 with the sequences of $C_{0}=\{166742\}$ and $C_{1}=\{166435\}$. The resulting simulated plot of the ISF is given in Fig. 2. To make the result more general, the horizontal axis is given in terms of the normalized frequency $f T$ (where $T$ is the interpulse period). Note that for very low frequency, the value of the ISF is about $-12.04 \mathrm{~dB}$, which is the result obtained earlier for a low-frequency interference signal. This is the minimum ISF (minimum in magnitude, which is the context implied in this paper). In order to obtain an analytical expression of the ISF for the case of sinusoidal interference signals, we may take this rather conservative value. The resulting $\mathrm{ISF}_{\min }$ is then given as

$$
\mathrm{ISF}_{\text {min }}=10 \log _{10}\left(\frac{N_{p q}^{2}}{4 N^{2}}\right)
$$

where

$$
N_{p q}=N_{p}+N_{q}
$$

with

$$
N_{p}=\left|\sum_{i=1}^{N} c_{i}\right|
$$

and

$$
N_{q}=\left|\sum_{i=1}^{N} c_{i}^{\prime}\right| .
$$

As $p$ and $q$ are fixed for any valid value of $N$ by the complementary property, hence, $N_{p}$ and $N_{q}$, as well as ISF ${ }_{\text {min }}$, are likewise fixed. Accordingly, it would be interesting to have codes, which would not vary substantially from complementary codes, but with the values of $p$ and $q$ equal to $N / 2$. Under this condition, $N_{p}$ and $N_{q}$ will have values of zero, and ISF $\min$ will have a value of $-\infty$, at least for the range where (23) is applicable. Codes having this characteristic do exist and are hereafter called subcomplementary codes. 


\section{SUBCOMPLEMENTARY CODES}

\section{A. Basic Properties}

1) Definition: A subcomplementary code pair, consisting of the sequences $C_{0}$ and $C_{1}$, whose elements are given by (1) and (2), respectively, has the property that the sum of the ACFs of the two sequences of the pair is equal to zero, except for the 0 and 1 lags, where it is equal to $2 N$ and $-N$, respectively, i.e.,

$$
R_{0}(\gamma)+R_{1}(\gamma)= \begin{cases}2 N, & \text { if } \gamma=0 \\ -N, & \text { if } \gamma= \pm 1 \\ 0, & \text { otherwise }\end{cases}
$$

where $R_{0}$ and $R_{1}$ are the ACFs of $C_{0}$ and $C_{1}$ and are given by (5) and (6), respectively. The difference between the complementary and the subcomplementary code pairs is that the latter has sidelobes at lag 1 . This is the price a user has to pay in order to get the advantage gained by the interference suppression capacity of the codes. This is not the first time that deviation from complementarity has been accepted to gain other performance parameters. Sulzer and Woodman [6] have also used quasi-complementary codes with nonzero sidelobes to minimize the effects of instrumental imperfections.

2) Autocorrelation Relationships: As mentioned earlier, for subcomplementary code pairs, $p$ and $q$ are both equal to $N / 2$. It is interesting to derive the magnitude of the sidelobe at lag 1 .

Using the definitions of even pairs and noting that $p$ and $q$ are equal to $N / 2$, it can be easily observed that each of the $p$ ones in sequence $C_{0}$ forms an odd pair with each of the $N-p$ of -1 's in the same sequence. The same is true with sequence $C_{1}$. Accordingly, the total number of odd pairs of all spacings in the two sequences is given by

$$
N_{o}^{T}=2 p(N-p)=\frac{N^{2}}{2} .
$$

The total number of pairs of all spacings in the two pairs is given by

$$
N_{o, e}^{T}=N(N-1) .
$$

Accordingly, the total number of even pairs of all spacings in the two sequences is given by

$$
N_{e}^{T}=N(N-1)-\frac{N^{2}}{2} \text {. }
$$

The total number of pairs with spacing of one of the two sequences is equal to

$$
N_{s p 1}^{T}=2(N-1)
$$

The total number of even and odd pairs with spacings of two and above $\left(N_{s p 2 a}^{T}\right)$ is obtained as

$$
N_{s p 2 a}^{T}=N^{2}-3 N+2 .
$$

As the complementary property is satisfied for these range of lags, then the total numbers of even and odd pairs here are equal and have the value half of $N_{s p 2 a}^{T}$ each. Now it is possible to find the expressions for the number of odd $\left(N_{s p 1 o}^{T}\right)$ and even $\left(N_{s p 1 e}^{T}\right)$ pairs with spacings of one as

$$
N_{s p 1 o}^{T}=\frac{(3 N-2)}{2}
$$

and

$$
N_{s p 1 e}^{T}=\frac{(N-2)}{2} .
$$

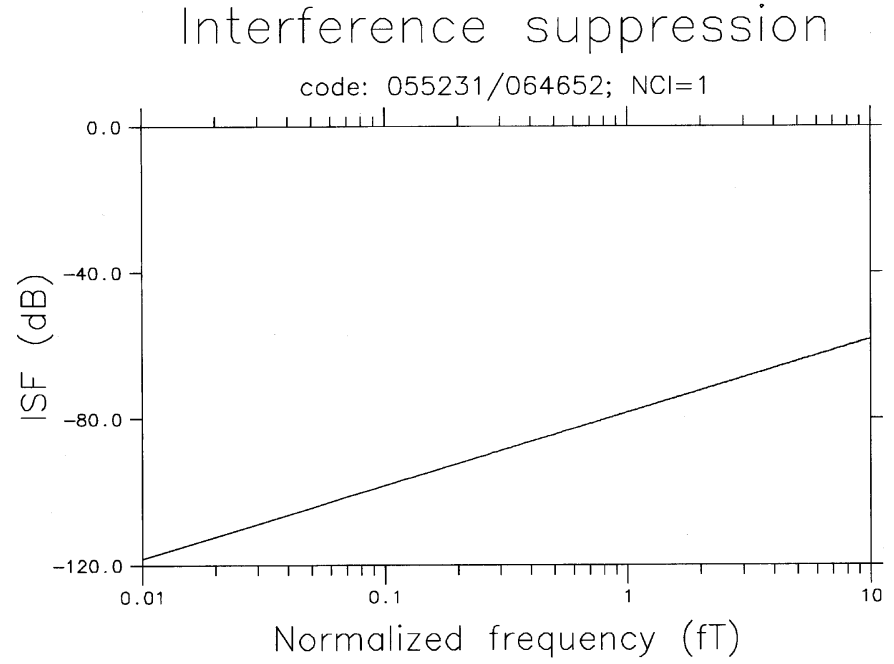

Fig. 3. ISF of subcomplementary code pair $\mathrm{CD}$ of length 16 with sequences of $C_{0}=\{055231\}$ and $C_{1}=\{064652\}$. The sequences are represented in octal format (see text for explanation)

The value of $R(1)$ is then given as $N_{s p 1 e}^{T}-N_{s p 1 o}^{T}$, which is equal to $-N$.

Although we have not proved it analytically yet, the values of the individual ACFs of the two sequences is observed in the already found code pairs to be equal to

$$
R_{0}(1)=1-\frac{N}{2}
$$

and

$$
R_{1}(1)=R_{0}(1)-2 .
$$

3) Permissible Values of $N$ : Equation (15) gives the necessary condition for complementarity. Using this relation, the values of $N$ for which complementary code pairs exist is determined as 2, 4, 8, 10,16, 20, 26, 32, etc. Although 18 also satisfies the condition, no pairs were found. Our objective here would be to determine which of the above lengths could have subcomplementary pairs. We must emphasize that this is limited to the above lengths only.

We consider either one of the two sequences. Let it be $C_{0}$. Since the number of pairs (even and odd) of this sequence with spacing of $\gamma$ is $N-\gamma$, then

$$
N_{o 0}(\gamma)+N_{e 0}(\gamma)=N-\gamma .
$$

Using (13) and rearranging, we have

$$
R_{0}(\gamma)=N-2 N_{o 0}(\gamma)-\gamma .
$$

Since $N$ is even, $R_{0}(\gamma)$ is even if $\gamma$ is even and odd for odd $\gamma$. That is, $R_{0}(\gamma)$ must be odd for $\gamma=1$. On the other hand, (35) and (36) must be satisfied. For this, $N / 2$ must be even. It follows then that subcomplementary code pairs do not exist for $N$ of 10 and 26. This obstacle is removed for these lengths, if we choose to place the sidelobe in lag 2 instead of lag 1 .

\section{B. ISF Characteristics}

As stated in Section II-B, the complementary code pairs having $N_{s}$ of zero give very high ISF performance, when the interference signal remains stationary for at least two interpulse periods (in absolute terms and taking the typical radar operating parameters into consideration, this is of the order of a few tens 

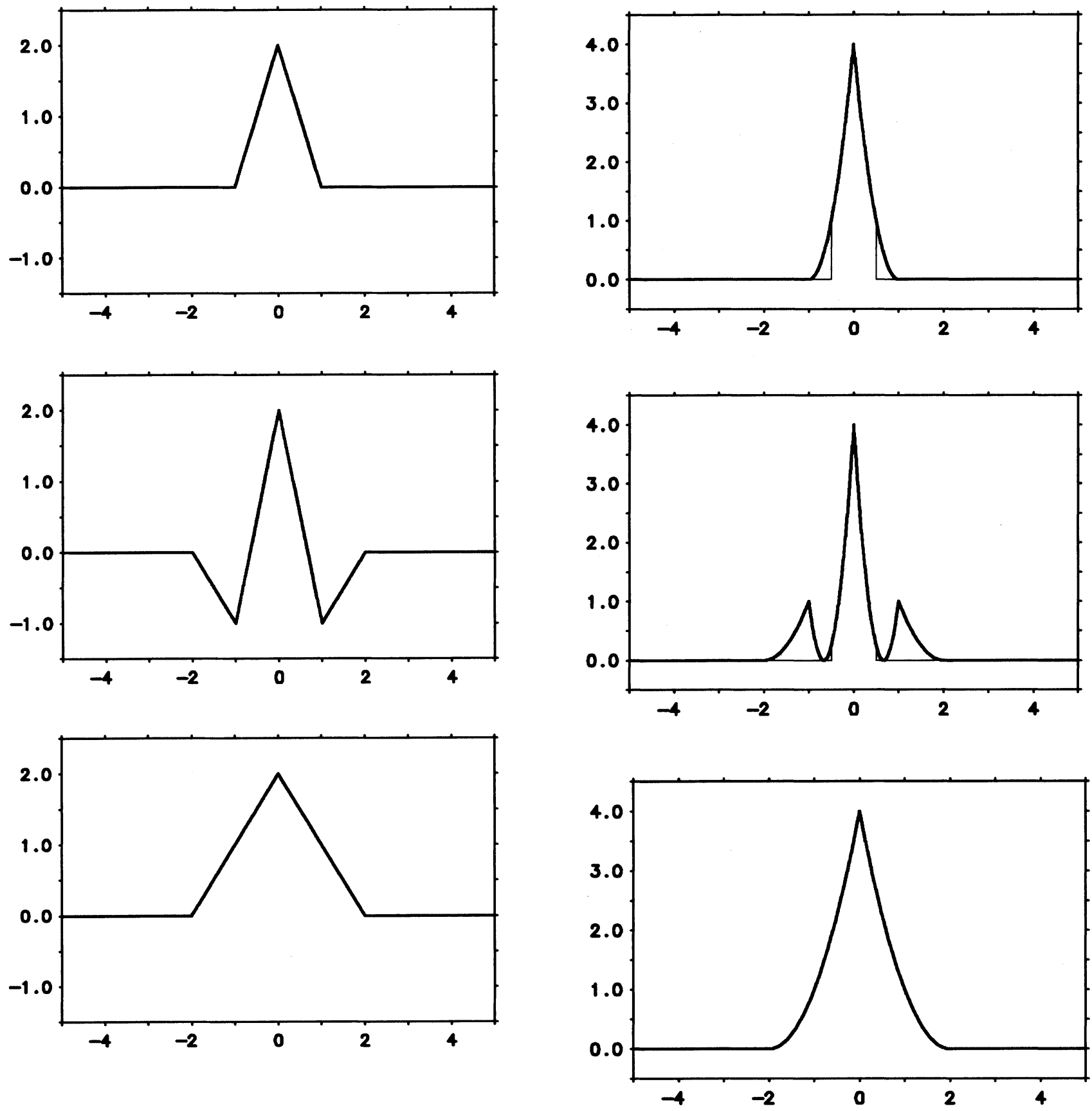

(a)

(b)

Fig. 4. (a) ACFs of a complementary code pair (top), a subcomplementary code pair (middle), and a complementary code pair having a pulse width double that of the first two cases (bottom), and (b) corresponding power values. For the sake of clarity, only the segment near-zero lag is shown, it being understood that the ACF values are zero for lags other than 0 and 1 . Note that the magnitudes shown are relative. Actual values are obtained by multiplying the ACF and power values by $N$ and $N^{2}$, respectively.

of hertz in and around the zero Doppler frequency). When this is not the case, then depending on the frequency spectrum of the interference signal and the code used, the interfering signal gives varying degrees of ISF performances. The minimum ISF possible is given by $\mathrm{ISF}_{\text {min }}$.

In order to extend the frequency range of effectiveness of the high ISF performance, it has been argued earlier that the number of ones and -1 's in each of the two sequences of a pair must be equal. In this case, high ISF performance is attained when the interference signal remains constant at least for the dura- tion of $N \tau$, where $\tau$ is the subpulse duration. Again, taking typical radar operating parameters, the range of effectiveness is extended from a few tens of hertz to a few tens of kilohertz. As an example, a plot of the ISF for a subcomplementary code pair of $N=16$ is given in Fig. 3. Comparing this with Fig. 2, the improvement in ISF performance is highly significant.

\section{Resolution Performance Considerations}

The main weakness of the subcomplementary code pairs is the presence of sidelobes at lag 1 . These sidelobes may be put 


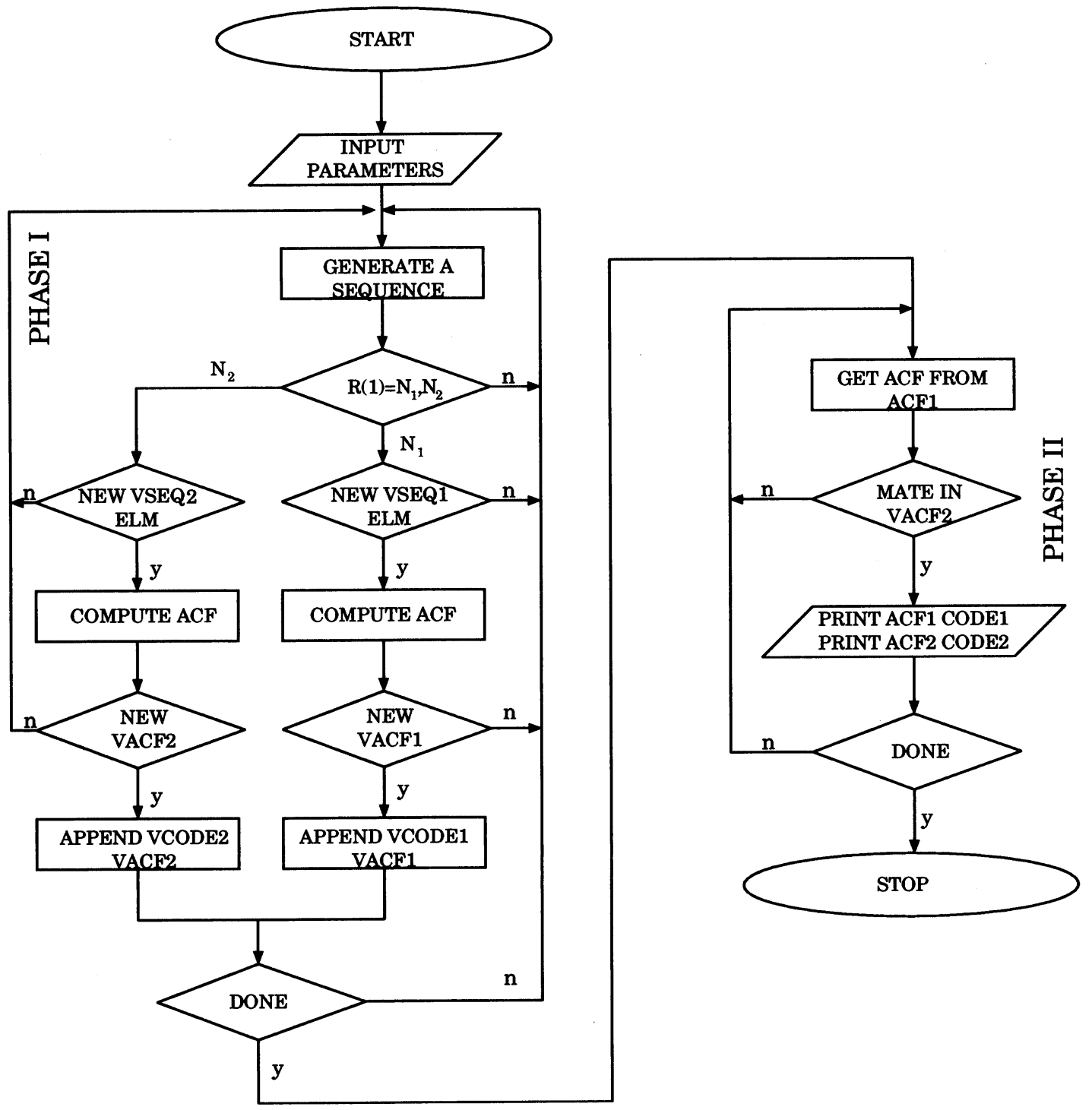

Fig. 5. Flowchart of the search algorithm. VSEQ1 and VSEQ2 are the vectors of sequences, while VACF1 and VACF2 are vectors of ACFs. The parameters to be input are the values of $N, N_{1}, N_{2}, T$, and COUNT.

TABLE I

List of SubComplementary CODE Pairs for Various Values of $N$. THe Elements of the Sequences ARe Given in Octal Notation (SEe TEXT FOR EXPLANATION)

\begin{tabular}{l|l|l|l|l}
\hline \hline$N$ & $C_{0}$ & $C_{1}$ & $R_{0}(1)$ & $R_{1}(1)$ \\
4 & 05 & 06 & -1 & -3 \\
8 & 246 & 251 & -3 & -5 \\
16 & 055231 & 064652 & -7 & -9 \\
20 & 1462532 & 1254551 & -9 & -11 \\
32 & 12626324531 & 23245262625 & -15 & -17 \\
\hline
\end{tabular}

at other lags, but it is felt that this is probably the best place. In order to give a better picture of this, the ACFs of complementary and the subcomplementary code pairs and their power distributions are shown in Fig. 4, where ideal signals and systems have
TABLE II

RADAR OPERATING PARAMETERS USED DURING THE EXPERIMENTAL OBSERVATIONS. CODE PAIR CD IS THE 16-BIT COMPLEMENTARY PAIR, AND Code Pair EF Is the 16-Bit SubComplementary Pair MENTIONED IN THE TEXT, WhILE THE UNCODED PULSE IS REPRESENTED BY NC. $N_{C I}$ AND $N_{I I}$ REPRESENT THE NUMBER OF COHERENT AND INCOHERENT INTEGRATIONS, RESPECTIVELY

\begin{tabular}{l|l|l|l|l}
\hline \hline Mode & IPP $(\mu s)$ & NFFT & $N_{C I}$ & $N_{I I}$ \\
\hline \hline NC & 400 & 128 & 38 & 5 \\
C-D & 400 & 128 & 19 & 5 \\
E-F & 400 & 128 & 19 & 5 \\
\hline
\end{tabular}

been assumed (i.e., the transmitted pulses are perfect rectangular pulses, and the receiver filter is ideal). Of course, in reality these are not attained, and the practical case is worse than what is given in Fig. 4 (e.g., see [23]). 


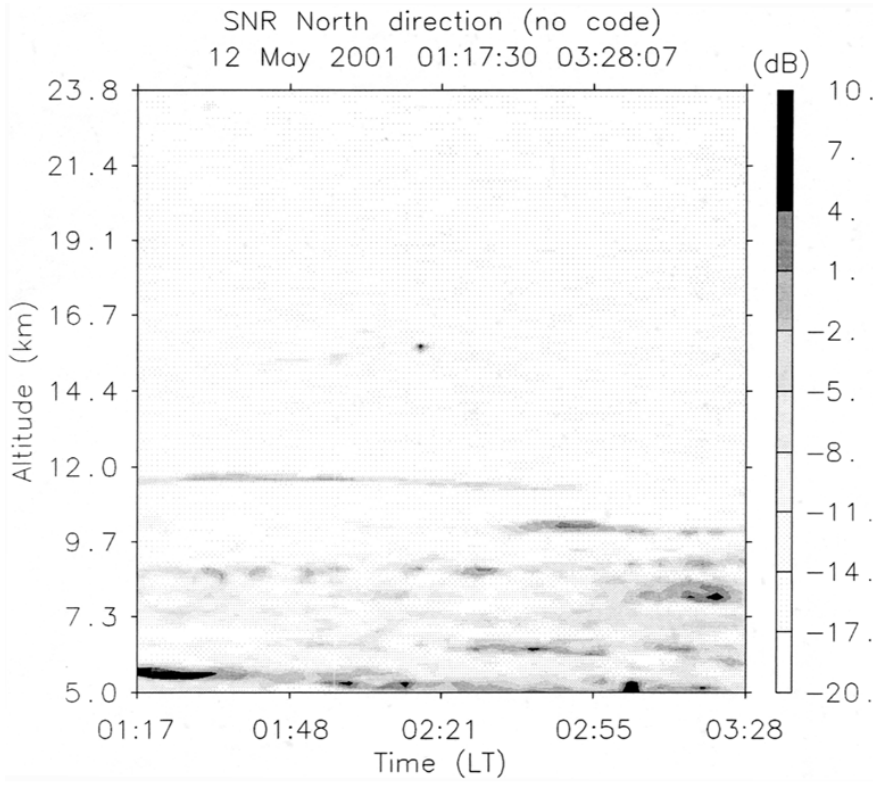

(a)

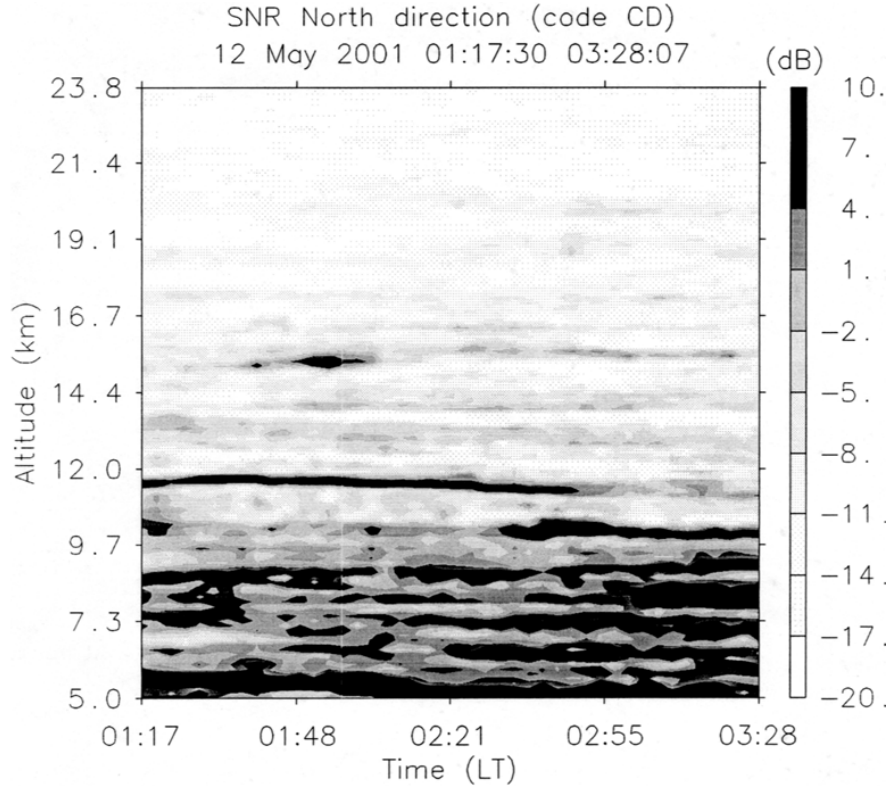

(b)

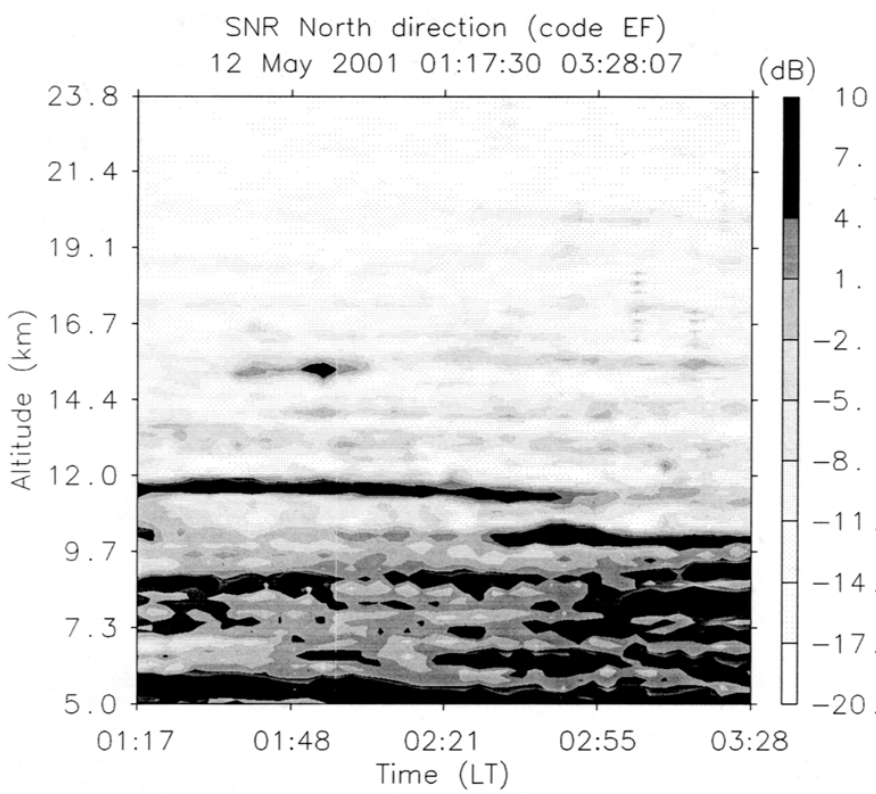

(c)

Fig. 6. (a) Range-time intensity plots of SNR profiles without pulse coding obtained in an oblique direction ( $10^{\circ}$ off zenith), (b) same as (a) using CD code, and (c) same as (a) using EF code.

It is to be noted that, even in the case of the complementary code pair, some of the decoded power is coming from neighboring range cells (the indicated areas). For comparison purposes, the case of a pulse having pulse width twice that of the original pulse is also shown in Fig. 4. Closer observation reveals that the worst case performance of the subcomplementary code pair is that of the reduced resolution of the wider pulse.

\section{Possible Applications}

When the effects of degradation in range resolution are acceptable, subcomplementary codes may be used in at least two cases of applications. In the first case, they may be used to suppress biases in and around zero Doppler frequency. In this frequency range, good complementary codes, or comple- mentary codes having $N_{s}$ of zero (see Section II-B) may also be used. However, subcomplementary codes are expected to perform better because they have higher ISF performance and greater frequency range of effectiveness (see Section III-B).

In the second case, subcomplementary codes may be used to suppress uncoded narrow-band interference signals having frequencies close to the radar center frequency. Such narrow-band interference signals may come from radio communication channels and other similar sources. In principle, this is not expected to occur as a result of appropriate frequency allocation. That is, one would not expect a radio communication channel to operate at the same frequency as the radar. In practice, this may happen, for example, in the lower very high frequency band during intense sporadic-E activities where interference signals from far 
away places may find their way to the radar system after being bounced by the E-region of the ionosphere.

In the case of a narrow-band interference signal close to the radar center frequency, it is unlikely that the frequencies of the interference and the radar signal be synchronized. As a result, the two frequencies are expected to drift from each other. Accordingly, subcomplementary codes are better placed to handle those type of signals than good complementary codes. On the other hand, it is important to note that the frequency range of effectiveness of subcomplementary codes constitutes a small fraction of the receiver filter bandwidth, and this may limit the scope of application of the codes.

\section{Search Technique}

In the absence of an analytical synthesis procedure, one possible way of generating subcomplementary code pairs for a given $N$ would be to consider the sequences having equal numbers of ones and -1 's and to look for pairs by performing tests for the subcomplementarity criteria. In view of the large number of sequences involved (there are $N ! /((N / 2) !(N / 2) !)$ sequences to be examined), this is only practically feasible using desktop computers, at best for $N$ up to 16 . To extend the search for $N$ up to 32, we have found out that the following technique, which is not exhaustive, may be used.

\section{A. Brief Description of the Technique}

The technique generates random sequences having equal numbers of ones and -1 's using the random-number generator of the computer system (the periodicity of the random-number generator should be checked [21]). Reference is made to Fig. 5, where a simplified flowchart of the algorithm is shown. At the heart of the technique is the GENERATE A SEQUENCE procedure. In effect, a vector for a sequence is defined; all its elements are set to -1 at the beginning; and half of them are then converted to ones using the index (position) obtained by

$$
I=1+\operatorname{INT}(N X)
$$

where $\operatorname{INT}(X)$ represents the integer part of $X$ and

$$
X=\operatorname{ran}(T)
$$

with $\operatorname{ran}(T)$ being the random-number generator, which returns a number between zero and one.

The task consists essentially of two phases. In the first phase, two sets of vectors are generated. The first set of vectors are the sequence vector VSEQ1, which stores the sequences whose $R(1)$ values are equal to $N 1$ [where $N 1=1-N / 2$; see (35)], and the corresponding vector for the ACFs VACF1. The second set of vectors are VSEQ2 and VACF2, and they are the counterparts of the first group of vectors for the sequences having $R(1)$ values equal to $N 2$ [where $N 2=N 1-2$; see (36)]. The objective of the first phase is then to get a specified number of elements for the two sets of vectors. The number of elements of the vectors of both groups $(\mathrm{COUNT}=\mathrm{COUNT} 1+\mathrm{COUNT} 2)$ to be obtained is set at the initialization stage. Here, COUNT1 represents the number of elements of VSEQ1 and VACF1, while COUNT2 is the corresponding value for VSEQ2 and VACF2. At the initialization stage, COUNT is entered, and COUNT1 and COUNT2 are determined by the program.

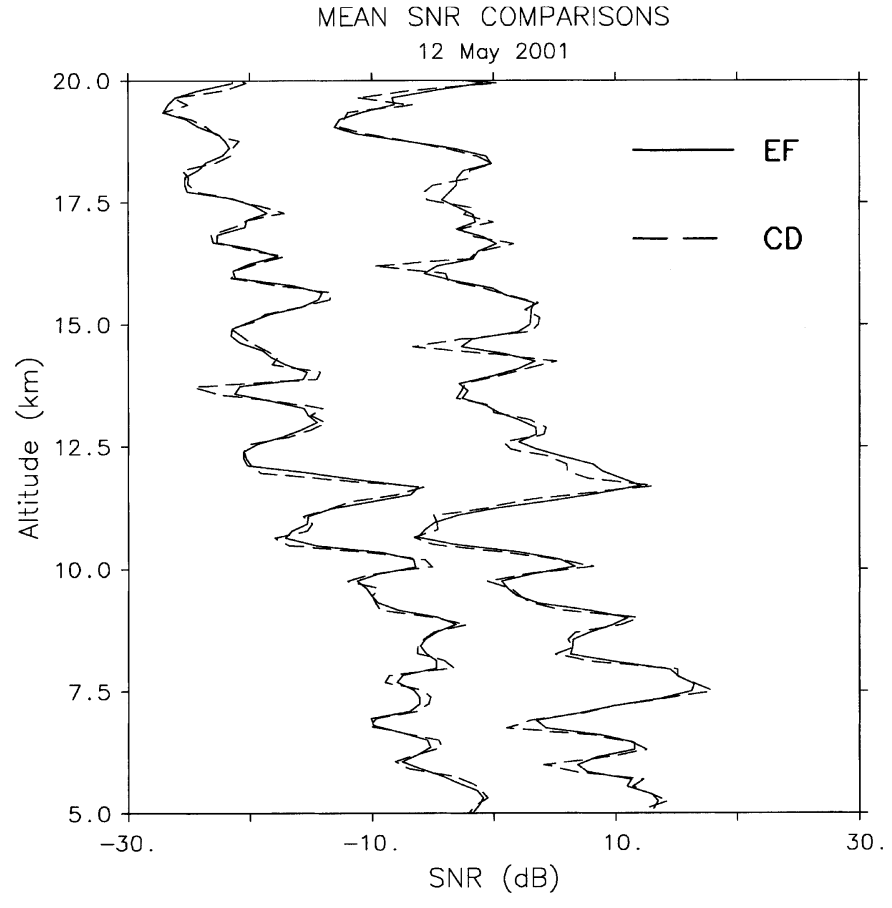

Fig. 7. Mean SNR profiles for the oblique (left) and the vertical (right) directions for the two coded modes. For the sake of clarity, the oblique profiles are displaced to the left by $10 \mathrm{~dB}$.

The second phase consists of searching for subcomplementary pairs, through the comparison of the autocorrelation functions. Each element of VACF1 is considered, one after the other, in comparison with the elements of VACF2. When there is a match, both the ACF values and the corresponding code sequences are printed.

The upper limit of the total number of elements of the vectors in the two groups is given by $N ! /((N / 2) !(N / 2) !)$. However, since every code sequence does not produce a unique $\mathrm{ACF}$, the number is much lower than that.

\section{B. Results of the Search}

The results obtained for $N$ of $4,8,16,20$, and 32 are shown in Table I. The values of the ACF for lag 1 of every sequence are also given. The code pairs given in Table I are those having the smallest value of sidelobe parameter (SP) defined as

$$
\mathrm{SP}=\sum_{i=1}^{N-1}|R(i)|
$$

where $R$ is the autocorrelation function of the sequence.

The time taken for the search is very short, up to $N$ of 16 , although some time is taken to find out the appropriate value of COUNT. For $N$ of 20, the time taken on a high-performance personal computer is of the order of $10 \mathrm{~min}$. For $N$ of 32, the time taken is of the order of five days.

\section{EXPERIMENTAL OBSERVATIONS}

Observations using the middle and upper atmosphere (MU) radar, located at Shigaraki in Japan [17]-[20] were conducted using three different modes in order to compare the respective range resolution performances using configurations similar to classical atmospheric observations performed by the radar. 

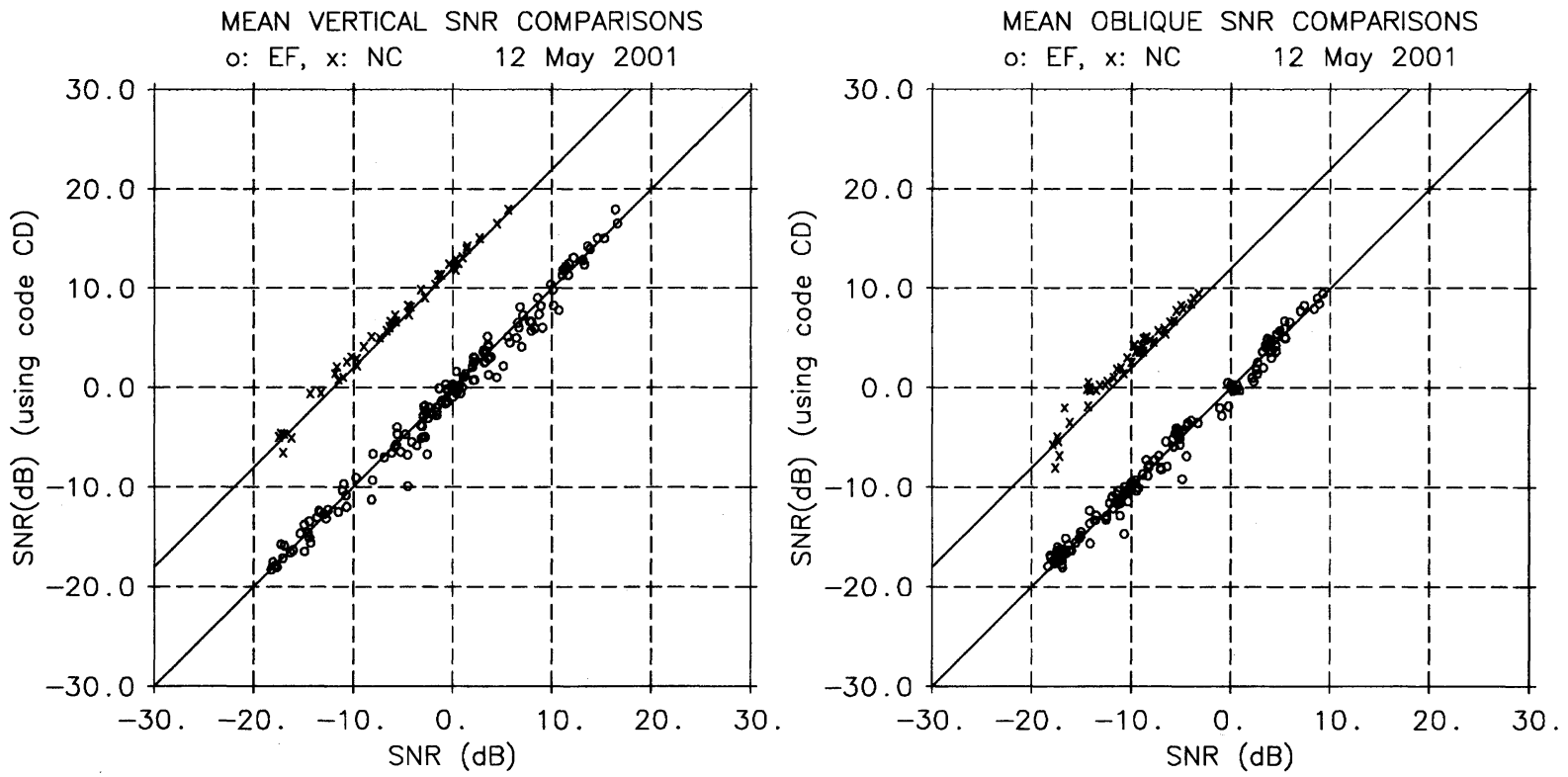

VERTICAL SNR COMPARISONS $0: E F, x: N C$ 12 May 2001
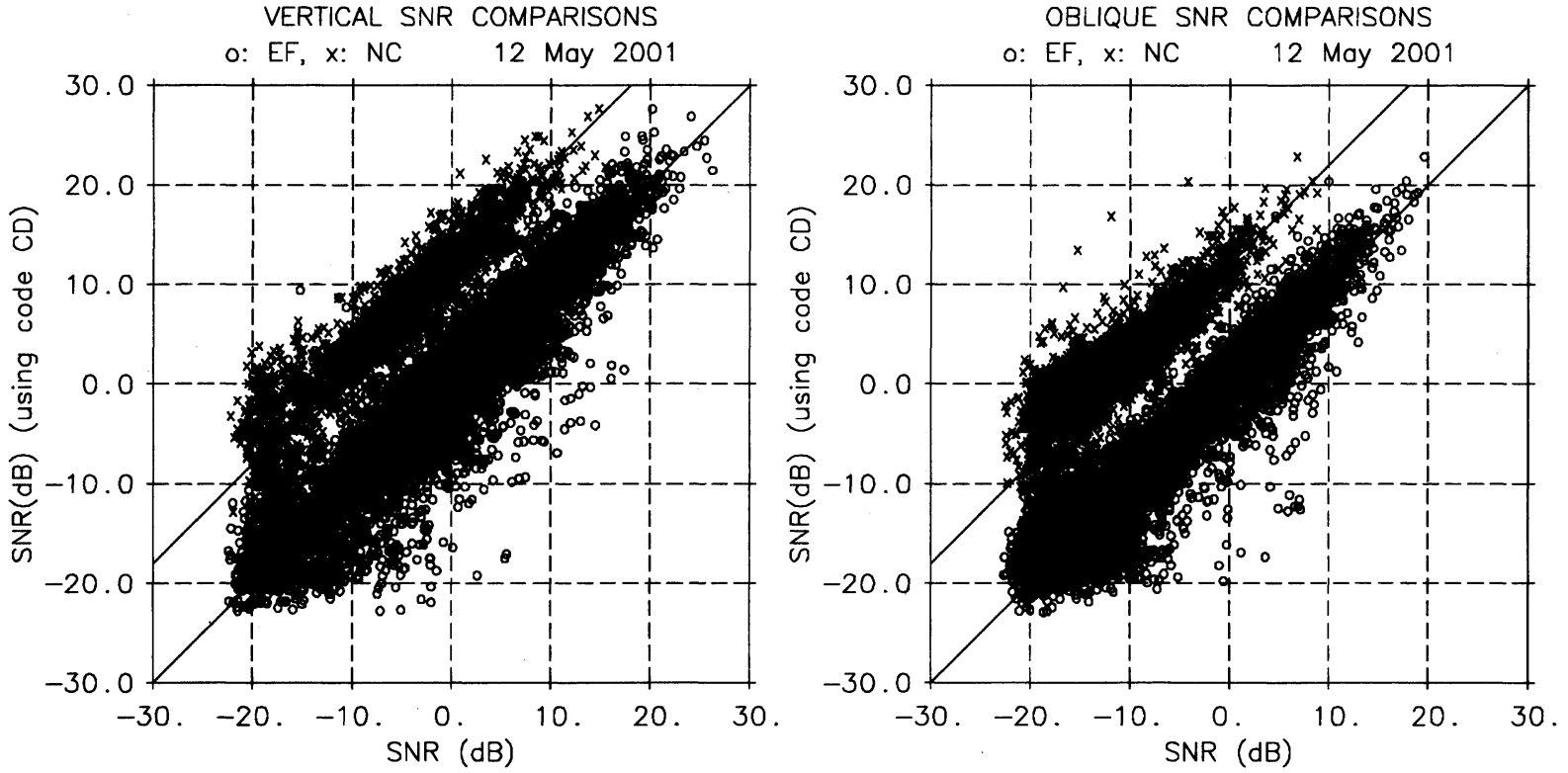

Fig. 8. SNR scatter diagrams for mode NC versus mode CD (upper line), and for mode EF versus mode CD (lower line), both for the vertical and oblique directions. The upper diagrams are for the mean values, while the lower ones are for all the data points.

\section{A. Experimental Configuration}

The radar was operated in a cyclic sequence using three modes of operation. These are 1) a uncoded $1-\mu$ s pulse, 2) a 16- $\mu$ s pulse coded with a 16-bit complementary code pair, and 3) a $16-\mu$ s pulse coded with a 16-bit subcomplementary code pair. The set of observation parameters used are given in Table II. The complementary code pair used is $\mathrm{CD}$ whose sequences are given by $C_{0}=051637$, and $C_{1}=003312$, and the subcomplementary code pair is EF, whose sequences are given by $C_{0}=055231$ and $C_{1}=064652$. The names for the code pairs have been arbitrarily given for reference purposes.

\section{B. Results of Observations}

The data collected during about two hours of observations enables us to make some comparisons of the performances of the new code pair $(\mathrm{EF})$ against those of the complementary code pair (CD) and the uncoded pulse (NC) in terms of resolution degradation in SNR, velocity, and related aspects. The details are given next.

1) SNR Comparisons: The evolution of the SNR during the observational period for the three modes NC, CD, and EF of operation are given in Fig. 6(a)-(c), respectively. The main structures are well identified in the three cases. There is a slight but noticeable loss of contrast of the profile for $\mathrm{EF}$ with reference to that of CD. As is well known, the enhancement of the SNR in the coded modes enables the identification of more structures, particularly at higher altitudes with respect to the uncoded mode.

Fig. 7 shows the mean signal-to-noise ratio (SNR) of the two coded modes (CD and $\mathrm{EF}$ ) for oblique and vertical directions. There is a high degree of similarity, but again, there are slight and noticeable differences between the two cases. The smoothing effect of the sidelobes of EF is clearly recognizable. 

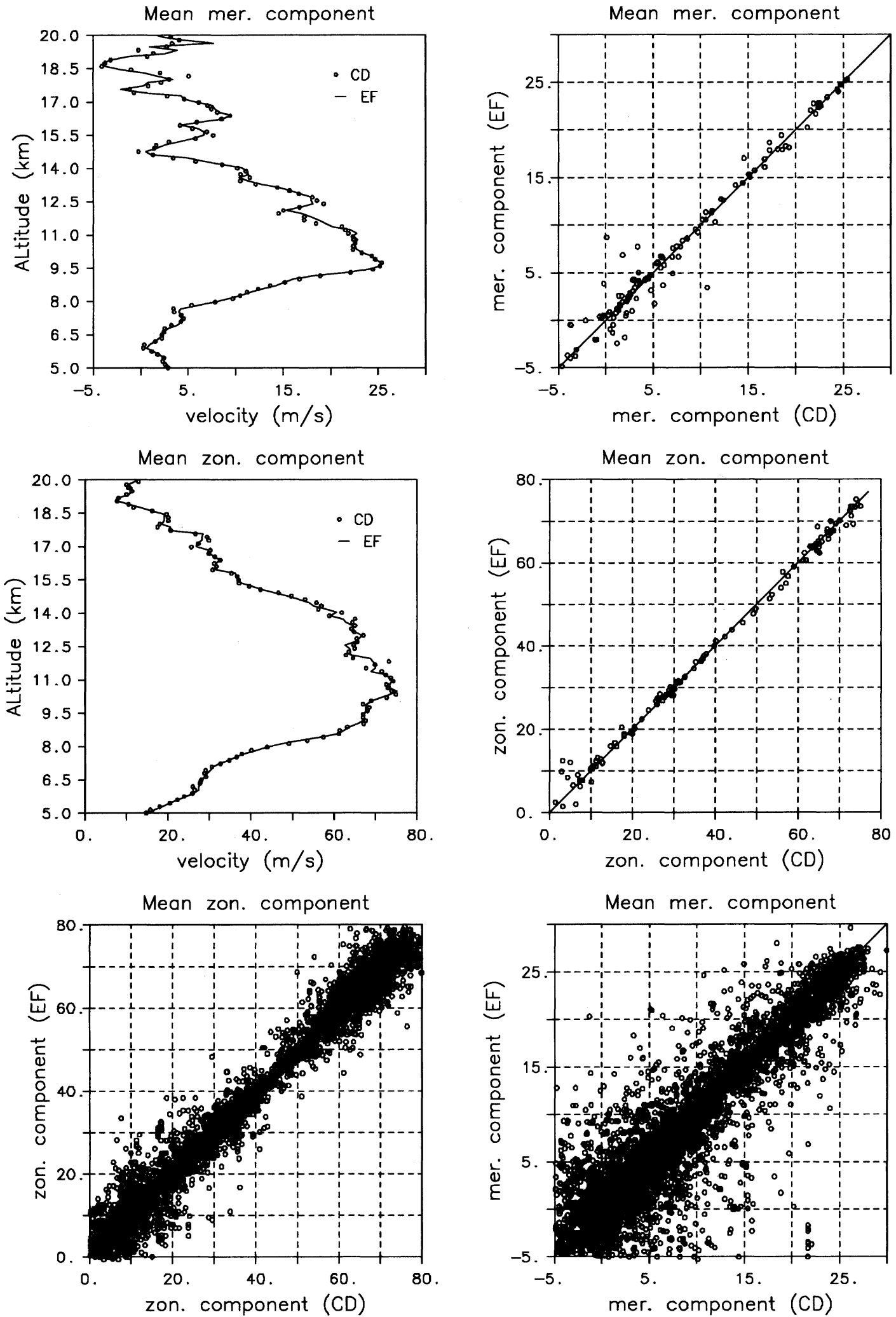

Fig. 9. Mean velocity profiles of the two code pairs for the meridional (top) and zonal (center) components of the wind along with their respective scatter diagrams. The bottom diagrams are for all the data points.

Scatter diagrams for the SNR values of the two codes are shown in Fig. 8. In all four diagrams, the upper line represents the values of $\mathrm{NC}$ versus $\mathrm{CD}$, while the lower line represents the corresponding values for $\mathrm{EF}$ versus $\mathrm{CD}$. SNR values below
$-20 \mathrm{~dB}$ have been rejected, as these are thought to be very likely due to noise rather than to signal. The scatter is slightly bigger for the case of EF versus CD. Accordingly, the degradation of the performance of code pair EF is really very small. The in- 

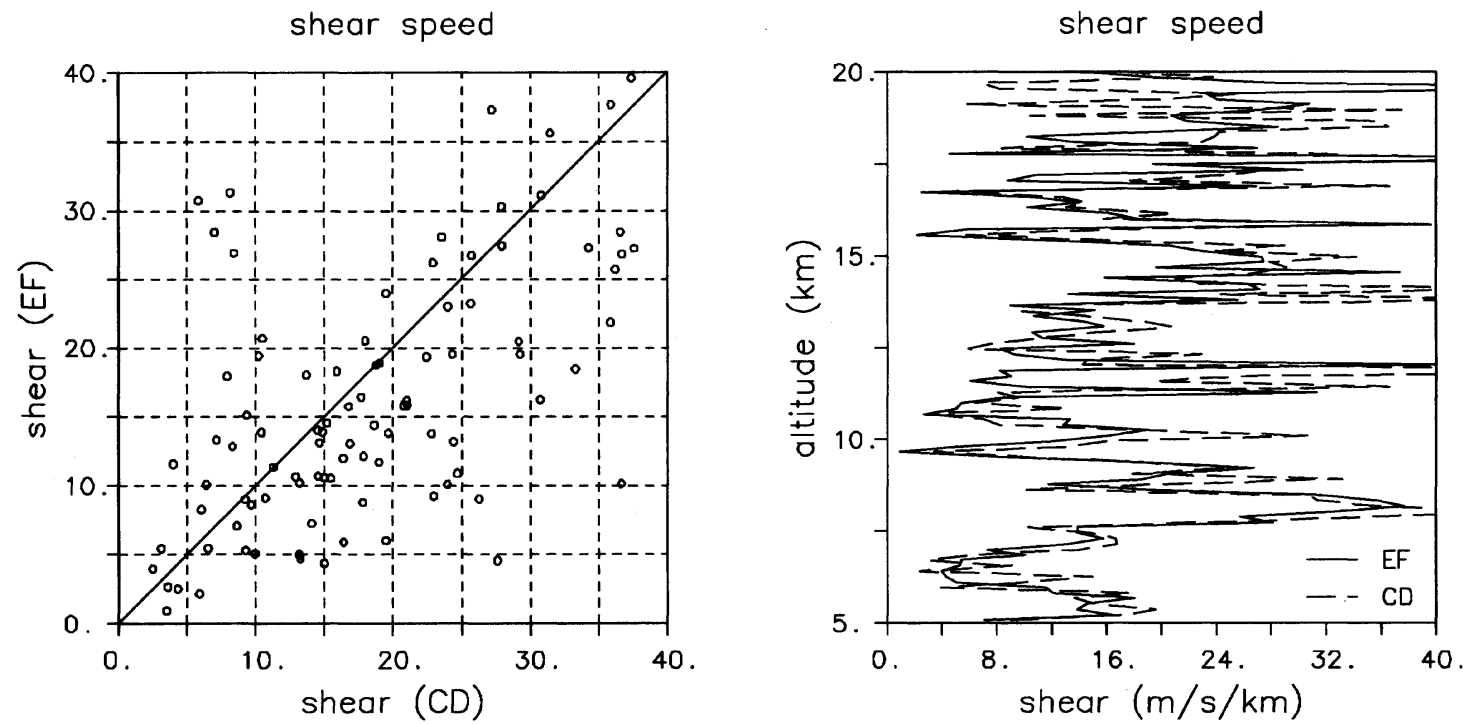

Fig. 10. Shear speed profiles of the two codes (right) and the corresponding scatter diagram (left) deduced from the wind observations.

crease in the scatter as the SNR decreases is noticeable and is attributed to the increase of estimation errors as SNR decreases. It is useful to mention here that a part of the observed spread of the scatter plot is also attributed to the variability in atmospheric reflectivities during the observation period.

2) Velocity Comparisons: In principle, the degradation in velocity resolution is expected to be smaller due to the nature of velocity estimation. This is indeed reflected in the results of the observations. Fig. 9 gives the mean values of the velocity profiles both for the meridional and zonal components of the wind together with the associated scatter diagrams. The correlation is very high, indicating very small degradation in performance. There is a noticeable increase in the scatter in the bottom left diagram of Fig. 9 for velocities between about $60-80 \mathrm{~m} / \mathrm{s}$. This is attributed to the decrease of SNR at this range of velocities.

3) Shear Comparisons: The shear profiles of the two codes are given in Fig. 10. These have been obtained from the wind profiles of Fig. 9 through differentiation. Good qualitative agreement in the height profiles is observed (e.g., see the positions of the extreme values). However, it is clearly observed that the scatter is greater here (as compared to the corresponding values for the velocity profiles), and the shear intensity deduced from observations using code pair CD is on the average greater than the corresponding value of code pair $\mathrm{EF}$ (mean values are 15.5 and $13.7 \mathrm{~m} / \mathrm{s} / \mathrm{km}$, respectively).

\section{CONCLUSION}

The simulation results show that subcomplementary code pairs have very high ISF values in and around zero Doppler frequency. In comparison with complementary code pairs with $N_{s}$ of zero, where the very high value of ISF is limited to a few tens of hertz around zero Doppler frequency, the corresponding value for the case of the subcomplementary code pairs is extended to a few tens of kilohertz (high values of ISF are obtained if the interference signal remains almost constant during a time period of the order of $N \tau$ for the case of subcomplementary codes, and during two interpulse periods in the case of complementary codes). This characteristic of the subcomplementary codes may be exploited to inhibit external interferences.

The main disadvantage of the subcomplementary codes is associated with the presence of the large sidelobes in the autocorrelation function at lag 1 . The sidelobes degrade the resolution performance of the codes. The results of the experimental observations have revealed this weakness. The performance degradation is dependent on the quantity to be estimated. The degradation in the estimation of wind is minimal. This is to be expected. The performance in shear wind speed is the worst. This is also to be expected. The degradation in performance in the estimation of the SNR falls in the middle. In comparing the performances of the subcomplementary code against the complementary code, it must be noted that part of the discrepancy could also be attributed to atmospheric variability during the observation period.

In view of the comparisons that have been made, it can be said that in the event where the advantage of interference suppression is gained, it may be reasonable to sacrifice degradation in performances in the estimation of wind and parameters derived from SNR observations. Even though the use of subcomplementary codes is not recommended for the study of the morphology of thin atmospheric layers such as those reported in [22], the performance degradation in SNR-related observations is not severe. The degradation is even insignificant in the case of wind measurements and may be considered negligible in some cases, e.g., in meteorological applications.

\section{ACKNOWLEDGMENT}

The assistance in computing facilities provided by H. Hashiguchi and S. Kawamura are gratefully acknowledged. R. Wiens read the entire manuscript and gave useful comments. The comments of the anonymous reviewer were also useful. The authors are grateful for both comments. 


\section{REFERENCES}

[1] G. Schmidt, R. Ruster, and P. Czechowsky, "Complementary codes and digital filtering for detection of weak VHF radar signals from the mesosphere," IEEE Trans. Geosci. Electron., vol. 17, pp. 154-161, 1979.

[2] R. F. Woodman, "High-altitude resolution stratospheric measurements with the Arecibo 430-MHz radar," Radio Sci., vol. 15, pp. 417-422, 1980.

[3] K. Wakasugi and S. Fukao, "Sidelobe properties of a complementary code used in MST radar observations," IEEE Trans. Geosci. Remote Sensing, vol. GES-23, pp. 57-59, Jan., 1985.

[4] O. Ghebrebrhan, "Sidelobe properties of complementary codes," Meteorol. Rdch., vol. 42, pp. 109-114, 1990.

[5] E. Spano and O. Ghebrebrhan, "Complementary sequences with high sidelobe suppression factors for ST/MST radar applications," IEEE Trans. Geosci. Remote Sensing, vol. 34, pp. 317-329, Mar. 1996.

[6] P. Sulzer and R. F. Woodman, "Quasi complementary codes: A new technique for MST radar sounding," Radio Sci., vol. 19, no. 1, pp. 337-344, 1984.

[7] E. Spano, "Optimum sets of complementary code pairs for ST/MST radar applications: Experimental results," in Proc. 7th MST Radar Workshop, Hilton Head Island, SC, 1996, pp. 193-200.

[8] O. Ghebrebrhan, "Full decoding of truncated ranges for ST/MST radar applications," IEEE Trans. Geosci. Remote Sensing, vol. 28, pp. 14-18, Jan. 1990.

[9] O. Ghebrebrhan and M. Crochet, "On full decoding of truncated ranges for ST/MST radar applications," IEEE Trans. Geosci. Remote Sensing, vol. 30, pp. 38-45, Jan. 1992.

[10] E. Spano and O. Ghebrebrhan, "Sequences of complementary codes for the optimum decoding of truncated ranges and high sidelobe suppression factors for ST/MST radar systems," IEEE Trans. Geosci. Remote Sensing, vol. 34, pp. 330-345, Mar. 1996.

[11] E. Spano, "Codage en phase et traitement des signaux en radarmétéorologie: étude théorique et réalization," Ph.D. dissertation, Univ. Toulon, Toulon, France, 1994.

[12] O. Ghebrebrhan, H. Luce, M. Yamamoto, and S. Fukao, "Interference suppression factor characteristics of complementary codes for ST/MST radar applications," Radio Sci., submitted for publication.

[13] M. J. E. Golay, "Complementary series," IRE Trans. Inform. Theory, vol. IT-7, pp. 82-87, 1961.

[14] L. R. Rabiner and B. Gold, Theory and Application of Digital Signal Processing. Englewood Cliffs, NJ: Prentice-Hall, 1975.

[15] E. Spano and O. Ghebrebrhan, "Pulse coding techniques for ST/MST radar systems: A general approach based on a matrix formulation," IEEE Trans. Geosci. Remote Sensing, vol. 34, pp. 304-316, Mar. 1996.

[16] O. Ghebrebrhan, E. Spano, and M. Crochet, "A search technique for complementary code pairs with good performances for the full decoding of truncated ranges," in Proc. 5th MST Radar Workshop, Aberystwyth, U.K., 1992, pp. 433-438.

[17] S. Fukao, T. Sato, T. Tsuda, S. Kato, K. Wakasugi, and T. Makihira, "The MU radar with an active phased array system, 1, antenna and power amplifiers," Radio Sci., vol. 20, no. 6, pp. 1155-1168, 1985.

[18] S. Fukao, T. Tsuda, T. Sato, S. Kato, K. Wakasugi, and T. Makihira, "The MU radar with an active phased array system, 2, in-house equipment," Radio Sci., vol. 20, no. 6, pp. 1169-1176, 1985.

[19] S. Fukao, T. Sato, T. Tsuda, M. Yamamoto, M. D. Yamanaka, and S. Kato, "Mu radar: New capabilities and system calibrations," Radio Sci., vol. 25, no. 4, pp. 477-485, 1990.

[20] M. Yamamoto, T. Nakamura, T. Tsuda, and S. Fukao, "The MU radar real-time data processing system," in Proc. 7th MST Radar Workshop, Hilton Head Island, SC, 1996, pp. 489-492.

[21] W. H. Press, S. A. Teukolsky, W. T. Vetterling, and B. P. Flannery, Numerical Recipes in FORTRAN 77: The Art of Scientific Computing, 2nd ed. Cambridge, U.K.: Cambridge Univ. Press, 1995.

[22] F. Dalaudier, C. Sidi, M. Crochet, and J. Vernin, "Direct evidence of sheets in the atmospheric temperature field," J. Atmos. Sci., vol. 51, pp. 237-248, 1994.

[23] D. S. Zrnic and R. J. Doviak, "Matched filter criteria and range weighting for weather radar," IEEE Trans. Aerosp. Electron. Syst., vol. AES-14, pp. 925-930, 1978

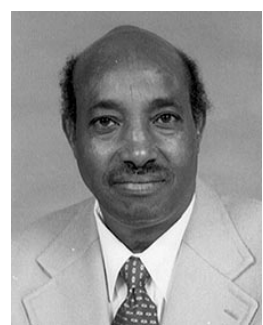

Ogubazghi Ghebrebrhan was born in Aykebetzu, Eritrea. He received the B.S. degree in electrical engineering from the Addis Ababa University, Asmara, Ethiopia, in 1977, the M.S. (D.E.A.) degree from the University of Nice, Nice, France, in 1982, and the Ph.D. (Dr.es.Sc.) degree from the University of Toulon, Toulon, France, in 1987.

$\mathrm{He}$ is currently heading the Geophysics Research Project of the University of Asmara, Asmara, Eritrea. He has been with the Geophysical Observatory of the Addis Ababa University from 1977 to 1992. In 1992, he joined the Department of Physics, University of Asmara. He has served as the Director of the Institute of Research and Development of the University of Asmara from October 1992 to March 1994, and as the Dean of the College of Science from March 1994 to November 2000.

Dr. Ghebrebrhan has been an Associate Member of the International Center of Theoretical Physics in Trieste, Italy, from 1991 to 1998.

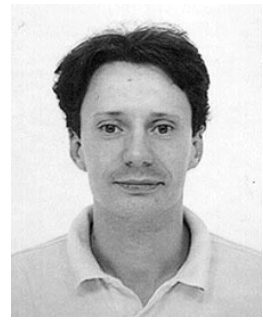

Hubert Luce was born near Lille, France. He received the Ph.D. degree in atmospheric radar techniques from the University of Toulon, Toulon, France, in 1996.

$\mathrm{He}$ is currently an Associate Professor at the University of Toulon, LSEET, France. He was a postdoctoral Lavoisier grant fellow of the French Ministery of Foreign Affairs from 1998 to 2000 and JSPS fellow of Monbusho from 2000 to 2002, both at the Radio Science Center for Space and Atmosphere, University of Kyoto, Japan.

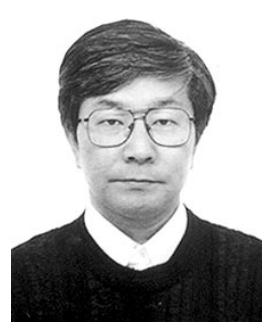

Mamoru Yamamoto received the B.E., M.E., and Ph.D. degrees in electronics from Kyoto University, Kyoto, Japan, in 1983, 1985, and 1988, respectively.

He has been an Associate Professor with the Radio Science Center for Space and Atmosphere (RASC), Kyoto University since 1996. He has been studying dynamics in the ionosphere and the atmosphere by using atmospheric radars. Study of the mid-latitude ionospheric irregularities is his current major. He has also been studying the technical aspect of the radar system and the data processing technique used for it. $\mathrm{He}$ is an important member of the research group that operates the MU radar of RASC and joins the project for the Equatorial Atmospheric Radar (EAR) in West Sumatra, Indonesia.

Dr. Yamamoto was awarded the Obayashi Prize in 1996 by the Society of Geomagnetism and Earth, Planetary and Space Sciences (SGEPSS)

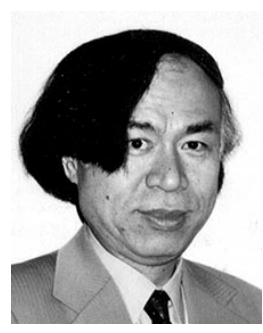

Shoichiro Fukao received the B.E., M.E., and Ph.D. degrees in electronics from Kyoto University, Kyoto, Japan, in 1967, 1969, and 1974, respectively.

$\mathrm{He}$ has been Professor of the Radio Science Center for Space and Atmosphere (RASC), Kyoto University since 1988, and has been Director of RASC from 1998 to 2002. His major is radar atmospheric physics, which includes radar-based research on dynamics and structure of the earth's atmosphere, radio-wave scattering, radar signal processing, and design and application of atmospheric radars. He is known for his contribution to the design and construction of the MU (Middle and Upper atmosphere) radar, a 50-MHz radar with an active-phased array system. In June 2001, he established another similar radar called Equatorial Atmosphere Radar (EAR) at the equator in West Sumatra, Indonesia. He has also developed small radars for lower-atmospheric observations. Its newest model (Lower Troposphere Radar; LTR) is currently in use as radars of the operational network of the Meteorological Agency of Japan (WINDAS).

Dr. Fukao has received several awards, including the NIKKEI Global Environmental Technology Prize in 1994 and the Shimadzu Prize in 2002. 